\title{
(Un)doing gender in organisationalen Rhythmen und Routinen
}

\author{
Julia C. Nentwich
}

\subsection{Bildung und Betreuung: Ideale guter Kinderbetreuung}

Gute Kinderbetreuung soll heute bilden und betreuen (Vogt 2015). Kitas übernehmen nicht nur die Betreuung der Kinder während der Berufstätigkeit der Eltern, sie haben auch einen Bildungsauftrag. Dies zeigt sich einerseits in Curricula, andererseits in Programmen und Qualitätsinitiativen. Im Orientierungsrahmen (Wustmann et al. 2012), einem gesamtschweizerischen Curriculum, werden Bildung, Erziehung und Betreuung als gleich wichtig und ineinander verzahnt dargestellt. Das Curriculum der Stadt Zürich für den Frühbereich nennt Erfahrungsfelder, die den Bildungsbereichen oder Fächern entsprechen (Walter-Laager 2015). Mit Programmen wie „infans“ (Andres und Laewen 2011) oder dem Netzwerk „Bildungskrippen“ (bildungskrippen.ch) werden Kinderbetreuerinnen und -betreuer darin weitergebildet, die Bildungsprozesse der Kinder zu dokumentieren. Das Qualitätslabel QualiKita setzt auf die Wichtigkeit von sowohl Betreuung als auch Bildung (QualiKita 2014).

Diese Entwicklung ist neu, Kindertagesstätten wurden die längste Zeit nahezu ausschließlich hinsichtlich ihrer Betreuungsaufgabe diskutiert. So zeigte eine Analyse des Mediendiskurses (Lanfranchi und Schrottmann 2004, S. 10), dass

Bei diesem Kapitel handelt es sich um eine aus dem Englischen übersetzte und grundlegend überarbeitete Fassung von Nentwich, Vogt \& Tennhoff (2016). Care and education? Exploring the gendered rhythms and routines of childcare work. In B. Liebig, B. Sauer, $\&$ K. Gottschall (Eds.), Gender equality in context: Policies and practices in Switzerland (S. 217-237).

J. C. Nentwich $(\varangle)$

Universität St. Gallen, St. Gallen, Schweiz

E-Mail: julia.nentwich@unisg.ch 
zumeist der gesellschaftliche und wirtschaftliche Nutzen der durch die Betreuung in der Kita ermöglichten Erwerbstätigkeit der Mütter betont wird und weniger der pädagogische Nutzen, der für die Kinder entsteht. Diese Betonung der Betreuung macht sich auch in den heutigen regulatorischen Anforderungen bemerkbar. Sowohl die nationalen gesetzlichen Grundlagen wie auch die vom Dachverband Kibesuisse empfohlenen Richtlinien zur Bewilligung einer Kita führen mehrheitlich Anforderungen an strukturelle Aspekte wie Raumgröße, Personalqualifikation und Mitarbeiter-Kinder-Verhältnis auf. Für die Genehmigung zum Betreiben einer Kindertagesstätte wird zwar auch ein pädagogisches Konzept benötigt, an dieses werden jedoch keine Anforderungen an den Inhalt und die pädagogische Qualität gestellt (Lanfranchi und Schrottmann 2004, S. 19; Faeh et al. 2021). Auch heute werden Kitas den Bildungszielen weniger gerecht als den erzieherischen Zielen, wie eine Untersuchung der Prozessqualität in 13 Schweizer Kitas (sowie in 16 Spielgruppen und sechs Tagesfamilien) gezeigt hat (Perren et al. 2016).

Mit dem Anspruch, sowohl zu betreuen als auch zu bilden, werden zwei wichtige Anforderungen an die Arbeit in Kitas formuliert und in die Berufs- und Organisationskultur eingeschrieben. Beide Zielsetzungen vermitteln zudem ein jeweils sehr spezifisches Verständnis davon, was unter guter Kinderbetreuung zu verstehen ist. Für unsere Analyse des hier stattfindenden doing und undoing gender ist dabei besonders relevant, dass mit diesen unterschiedlichen und sogar widersprüchlichen Bildern guter Kinderbetreuung jeweils auch das Geschlecht des Berufs und der damit verbundenen Tätigkeiten sowie damit einhergehende Vorstellungen von Weiblichkeit und Männlichkeit verhandelt werden.

Bereits die im 19. Jahrhundert und im Anschluss an die „Entdeckung“ der Kindheit als vom Erwachsenenleben abgrenzbarer Lebensphase mit ganz eigenen Bedürfnissen (Ariès 1998) entstandenen Anfänge der Kindertagesstätte als Institution sind eng mit den sich zur gleichen Zeit etablierenden bürgerlichen Geschlechtsvorstellungen verknüpft (Maihofer 2004). In den durch Fröbel entwickelten ersten Ansätzen frühkindlicher Bildung bezeichnet er die Kita als erste Stufe des bürgerlichen Bildungssystems (Rabe-Kleberg 2003). Der ebenfalls von Fröbel entworfene Beruf des frühkindlichen Erziehers ist klar als Männerberuf konzipiert und die ersten Kitas werden auch durch Männer geleitet (Aigner und Rohrmann 2012, S. 42; Mayer 2009, S. 200; Rabe-Kleberg 2003). Das Unterrichten wird von ihm als eine dem bürgerlichen Mann vorbehaltene Tätigkeit entworfen und explizit mit hegemonialen Männlichkeitsvorstellungen verknüpft. Allerdings setzt sich Fröbels Sichtweise nicht durch. Unter Betonung der besonderen Eignung von Frauen gerade für die Erziehungsberufe in der frühen Kindheit wird der Beruf zu einem der ersten Frauenberufe (Rabe-Kleberg 2003). Mit dem Ideal der Kinderbetreuerin als „fürsorgliche Mutter“ (Friis 2008, S. 23) rücken 
Aspekte der Ernährung und Hygiene ins Zentrum und das Ideal der guten Kinderbetreuung wird folglich mit Vorstellungen von Weiblichkeit und Mütterlichkeit verknüpft. Diese Konzeption wird nicht nur in Bezug auf den Beruf der Erzieherin, sondern auch hinsichtlich der Funktion und Legitimation der Institution selbst auf das Idealbild der Mutter in der bürgerlichen Familienvorstellung des 19. Jahrhunderts bezogen. So sind Schweizer Kindertagesstätten zunächst als ein Ersatz für die Betreuung durch die Mutter zu Hause konzipiert worden. Mit der Einführung von Kindergärten, Kindertagesstätten (Bewahranstalten) oder Vorschulen (Kleinkinderschulen) im 19. Jahrhundert (Roßbach und Grell 2012, S. 333) stand oftmals die Versorgung für Kinder aus Arbeiterfamilien im Mittelpunkt, da beide Elternteile in Fabriken arbeiteten (Falk 2018). Vor dem normativen Hintergrund des bürgerlichen Familienmodells galten Einrichtungen der frühkindlichen Bildung und Betreuung als das geringere Übel, wurde doch die Betreuung durch die Mutter in der Familie als das eigentlich zu realisierende Ideal gesehen.

Während die hausarbeitsnahe, mütterliche Tätigkeit der Betreuerin weiblich konnotiert wird, wird die frühkindliche Bildung durch männliche Lehrer ausgeführt. Betreuung und Bildung werden hierdurch entlang der bürgerlichen Vorstellung von Geschlecht als sich gegenseitig ausschließend aufgeladen. Wie wir an anderer Stelle ausführen (Kap. 6), wirkt sich die nach wie vor stabile Verbindung von Betreuung und Weiblichkeit auch heute noch unmittelbar auf die Positionen von Männern und Frauen aus, die in diesem Bereich arbeiten. Insbesondere männliche Kinderbetreuer müssen sich mit der im Idealbild der guten Kinderbetreuung enthaltenen Weiblichkeit auseinandersetzen (Nentwich et al. 2013; Cross und Bagilhole 2002).

In diesem Kapitel interessieren wir uns für einen organisationskulturellen Blick auf gute Kinderbetreuung aus einer praxistheoretischen Perspektive. Wir zeigen, wie die Alltagspraktiken der Kinderbetreuung entweder Aspekte der Betreuung oder aber der Bildung aktualisieren und so verschiedene Vorstellungen guter Kinderbetreuung konstruieren, indem sie mit Bezug auf den Betreuungsdiskurs das Idealbild der guten Mutter und Hausfrau und damit Weiblichkeit oder aber durch Aktualisierung des Bildungsdiskurses das Idealbild der guten Pädagogin bzw. des guten Pädagogen und damit ein geschlechtlich weniger klar festgelegtes Bild zeichnen. Wir untersuchen das hierbei stattfindende doing und undoing gender mit einer praxistheoretischen Perspektive auf die in den Arbeitsalltag eingeschriebene Rhythmen und Routinen, durch die jeweils das eine oder aber das andere Idealbild aktualisiert wird. 


\subsection{Doing und undoing gender in Rhythmen und Routinen}

Idealbilder beruflicher Praktiken sind durch Acker (1990) als zentrale Mechanismen der gendered organization, der vergeschlechtlichten Organisation, beschrieben worden. Wie Forschung zu männerdominierten Berufsfeldern gezeigt hat, beinhaltet das Bild des ideal worker, das Idealbild eines Mitarbeiters, jeweils bestimmte Annahmen über Körper, Biografie wie auch den Lebensstil. Bisher wurde es insbesondere im Management untersucht, wo das Ideal eng mit Vorstellungen von Männlichkeit und einer geschlechterdifferenzierenden Arbeitsteilung verknüpft ist (zum Beispiel Kelan 2008; Meriläinen et al. 2004). Auch Kinderkrippen, Kitas und Kindergärten gelten als gendered organisations; sie sind von Vorstellungen von Weiblichkeit geprägt und den dort vorgefundenen Idealbildern liegen stereotype Bilder von Weiblichkeit zugrunde (Sargent 2005; Cameron 2006; Warin 2006; Friis 2008). Insbesondere der mit Mütterlichkeit verknüpfte Betreuungsdiskurs ist eng mit dem Berufsbild verwoben (Sargent 2005). So stellen zum Beispiel Timmerman und Schreuder (2008) fest, dass der Betreuungsdiskurs nicht nur dominant ist (Vogt 2002a), sondern auch den Diskursen der Bildung und pädagogischen Orientierung widersprechend gegenübergestellt wird. Eine Verknüpfung, die der angestrebten Professionalisierung entgegensteht bzw. sie sogar verunmöglichen kann (Friebertshäuser 1997). Zugespitzt drücken dies Rabe-Kleberg und Rohrmann aus, wenn sie Kindergärten als „,weibliche Arenen“ (Rabe-Kleberg 2003, S. 64) bzw. als „Gärten der Frauen“ (Rohrmann 2009, S. 34) bezeichnen.

Worin zeigt sich diese Kultur der Weiblichkeit und in welchem Zusammenhang steht sie mit dem Bild der guten Kleinkindbetreuerin bzw. des guten Kleinkindbetreuers? Neben Symbolen, Artefakten und Routinen ist insbesondere die zeitliche Strukturierung des Arbeitsalltags als wichtiger organisationskultureller Aspekt anzusehen (Gherardi 1995). Aktuelle Studien zur Arbeitszeit zeigen, dass die unbegrenzte zeitliche Verfügbarkeit sowie Anwesenheit am Arbeitsplatz ein wichtiges Element zur Herstellung männlicher professioneller Identität darstellt (Gascoigne et al. 2015, S. 461). Es ist wiederum das bürgerliche Geschlechtsmodell mit seinen Annahmen zur geschlechterdifferenzierenden Arbeitsteilung, das sich hier eingeschrieben hat: Der ideal worker ist hier männlich und priorisiert die Arbeit gegenüber den privaten Verpflichtungen. Werden dem Ideal entsprechend zum Beispiel viele Überstunden gemacht, können Männer eine männliche, professionelle Identität aktualisieren, es findet doing gender statt. Zugleich wird so aber auch kommuniziert, was nicht dem Ideal entspricht und was das Andere in diesem Kontext ist (Godfrey et al. 2012). Doing gender bedeutet hier, dass 
Wissen über Geschlecht gemeinsam mit dem Wissen über eine bestimmte Profession oder Tätigkeit aktualisiert wird. Es handelt sich um miteinander verschränkte Praktiken, die sich gegenseitig herstellen und damit auch stabilisieren (Bruni et al. 2005).

Praxistheoretische Forschung hat gezeigt, wie die zeitliche Strukturierung des Arbeitsalltags einen Rhythmus produziert, durch den die kulturellen Normen und auch das Geschlechterwissen auf implizite Weise sowohl etabliert als auch reproduziert werden (Orlikowski und Yates 2002). Sie werden zu selbstverständlich gehaltenen und unhinterfragten Routinen, indem sie im Arbeitsalltag strukturierend etabliert und auch eingefordert werden. Rhythmen und Routinen können dabei immer auch gendered practices sein (Martin 2003), durch die situativ Männlichkeit und Weiblichkeit inszeniert und damit doing gender stattfinden kann. Auch wenn die situativen gendering practices äußerst flüssig und fluide sind und sowohl als doing als auch als undoing realisiert werden können, ermöglichen sie die konzeptionelle Verbindung von situativem Handeln mit institutionellem Wissen und betonen damit diskursanalytische Aspekte ethnografischer Forschung. (Un)doing gender kann so über eine Analyse der Idealbilder sowie ihrer Verwendung in Alltagspraktiken untersucht werden. Empirisch ist somit der Frage nachzugehen, wie die Idealbilder der guten Kinderbetreuung in gendered practices und damit in den alltäglichen Rhythmen und Routinen der Kita eingeschrieben sind und welches Wissen über die Profession sowie über Geschlecht in den Alltagspraktiken eines practicing gender jeweils aktualisiert wird.

\subsection{Rhythmen und Routinen ethnografisch untersuchen}

Zentral für die Analyse von Idealbildern und organisationalen Rhythmen und Routinen ist ein ethnografischer Zugang. Es muss sowohl das doing als auch das saying im fraglichen Kontext untersucht werden können (Bruni et al. 2005). Mit unserem ethnografischen Forschungsdesign (Eberle und Maeder 2010; Gherardi 2019; Gobo 2008) verbinden wir mehrere qualitative Forschungsmethoden: Interviews mit den Kitaleitungen sowie den männlichen und weiblichen Kinderbetreuenden, videogestützte Beobachtungen und job shadowing, die auch Ad-hoc-Interviews mit den Betreuungspersonen im Arbeitsalltag umfassten, ergänzt um Beobachtungen und Gespräche ohne Videokamera. Durch diese Verbindung von Beobachtung und Interview, von Interpretation des Gesehenen mit Diskurs- und Positionierungsanalyse wurde es möglich, das Prozesshafte, Interaktive und Fluide des (un)doing gender greifbar zu machen. 
Nach dem Verfahren des theoretischen Samplings (Glaser und Strauss 1967) haben wir aus den insgesamt 20 besuchten Kitas vier Einrichtung ausgesucht, in denen zum einen ausgebildete männliche Fachpersonen arbeiteten, die sich jedoch hinsichtlich des Männeranteils, des Raumkonzepts und der Trägerschaft unterschieden. Während in den Kitas Gelb, Rot und Grün je ein Mann mit abgeschlossener Ausbildung arbeitete, war das Verhältnis zwischen Männern und Frauen in Kita Blau ausgewogen. Hier arbeiteten zum Zeitpunkt unserer Untersuchung vier ausgebildete Männer und fünf ausgebildete Frauen. Ein wichtiges Kriterium für die hier vollzogene Analyse war das pädagogische Konzept. Hier nahmen wir an, dass sich die Diskurse Betreuung und Bildung auf unterschiedliche Weise zeigen sollten. Während die Kitas Blau und Gelb nur sehr kurze Angaben auf ihren Webseiten gemacht haben, sind die Konzepte der Kitas Rot und Grün weiter ausgearbeitet und eine spezifische pädagogische Orientierung ist expliziert.

Jeweils zwei Forscherinnen unseres Teams haben insgesamt 15 Tage in diesen vier Kindertagesstätten verbracht und den Arbeitsalltag beobachtet. Videounterstützt folgten wir im Sinn eines job shadowing (Czarniawska 2007) jeweils einer männlichen Fachperson; zudem beobachteten wir auch frei und machten dabei ausführliche Feldnotizen. Im Anschluss an jeden in der Kita verbrachten Tag diskutierten und teilten wir unsere Notizen miteinander und schrieben kurze Memos. Systematisch protokollierten wir für jeden Tag die jeweils anwesenden Personen sowie den genauen Tagesablauf mit den jeweils durch die Betreuenden ausgeführten Tätigkeiten und den Aktivitäten der Kinder. Diese Protokolle dienten zunächst dem allgemeinen Überblick über die beobachteten Szenen und sollten es zum Beispiel erleichtern, bestimmte Sequenzen in den Videoaufnahmen einfacher wiederfinden zu können. Bereits im Vergleich dieser Protokolle zwischen den vier beobachteten Kitagruppen fielen uns jedoch im Team große Unterschiede in der zeitlichen Dauer wie auch hinsichtlich einzelner Abläufe auf und weckten damit unser weiteres Interesse.

Eine praxistheoretische Analyse von (un)doing gender untersucht sowohl die Verwendung als auch die Nichtverwendung und auch den Widerstand gegenüber spezifischen Idealbildern in den alltäglichen Situationen des Arbeitsalltags. Insbesondere die Erwartungen und Geschichten über enttäuschte Erwartungen gelten als empirische Marker für ein mögliches undoing gender (Hirschauer 2001, S. 214ff.) und traten hier insbesondere im systematischen Vergleich der Beobachtungen aus vier verschiedenen Kitagruppen zutage. Weitere wichtige Datenquellen waren zudem die vielfältigen Interviewpassagen, in denen besonders die von uns interviewten männlichen Kinderbetreuenden über ihre häufig erlebten Herausforderungen mit der Kita-Kultur berichteten. Weiter entwickelten sich Gespräche 
in situ, wenn uns während einer beobachteten Sequenz etwas als erklärungsbedürftig auffiel. Für die hier vorliegende Analyse des (un)doing gender in den Alltagspraktiken der Kita analysierten wir primär die ethnografischen Feldnotizen und die im Rahmen der Ethnografie entstandenen Interviews. Das Material aus der Videoanalyse sowie die Interviews mit den Kinderbetreuerinnen und Kinderbetreuern zogen wir unterstützend hinzu. Dank des Videomaterials war es möglich, einzelne in den Feldnotizen beschriebenen Szenen nochmals zu schauen und auch verbatim Transkripte der darin enthaltenen Interaktionen und Dialoge für die Analyse bereitzustellen. Durch kontrastierendes Lesen der zentralen Szenen aus den vier Kindertagesstätten und dem gemeinsamen Austausch über unsere Interpretationen im Team entwickelten wir ,dichte Beschreibungen“ (Geertz 1977) der organisationalen Rhythmen und Routinen.

\subsection{Gendered practices? Rhythmen und Routinen der guten Kinderbetreuung}

Aspekte der Bildung und Betreuung wurden in allen von uns untersuchten Kitas verhandelt. Sowohl die Interviews mit Kitaleitungen als auch unsere Inhaltsanalyse der pädagogischen Konzepte aller 20 Kitas zeigen, dass, auch wenn Bildung in den letzten Jahren zu einem wichtigen Diskurs für Schweizer Kitas geworden ist, es in erster Linie der Diskurs der Betreuung ist, der klar im KitaAlltag verankert ist. Häufig wird in den Konzepten eine Tagesstruktur entlang der Mahlzeiten abgebildet, pädagogische Ziele nehmen neben der gesunden Ernährung und dem explizit erwähnten täglichen Spaziergang an der frischen Luft einen überraschend kleinen Stellenwert ein. Werden pädagogische Ziele und Vorstellungen von guter Erziehung und Bildung formuliert, bleiben diese häufig allgemein und vage formuliert, es fehlt weiterhin bei nahezu allen Dokumenten die Beschreibung konkreter Schritte im Alltag, die zur Zielerreichung notwendig wären. Insbesondere die Rolle des pädagogischen Personals für Bildungs- und Erziehungsprozesse bleibt unklar. Insgesamt scheinen Aspekte der guten Betreuung, wie zum Beispiel Hygiene, Sicherheit und Ernährung, stärker im Zentrum zu stehen.

Dass Aspekten der guten Betreuung eine größere Wichtigkeit zukommt bzw. diese als selbstverständlich angenommen werden, zeigen auch vielfältige Interviewpassagen mit männlichen Kinderbetreuern, in denen sie ihre erlebten Herausforderungen mit der Kita-Kultur beschreiben. So sensibilisierten uns verschiedene Erzählungen Retos zur Entwicklung des Waldkonzepts seiner Waldgruppe für die Wichtigkeit der Einhaltung der Hygiene in der Kita. In einem Ad-hoc-Interview 
im Wald beschreibt er ausführlich, wie er ein Hygienekonzept entwickelt habe, dieses jedoch auch an die Möglichkeiten im Wald habe anpassen müssen. Insbesondere die Ausführlichkeit seiner Erklärungen und Legitimationen für die entwickelte Praxis deuten auf die erlebten Widerstände hin. Das folgende Zitat gibt diese Reibungspunkte in seinen eigenen Worten wieder:

Von der Kita ist es immer so: Sicherheit, Kontrolle, immer wissen, wer wo ist, klare Regeln, ja, es ist einfach irgendwie, vielleicht widerspricht mir das einfach vom Charakter her. (Video-Memo Kita Grün)

Auch in den von uns ethnografisch näher untersuchten vier Kitas zeigte sich die Relevanz von Betreuung und Bildung, allerdings in je zwei der Kita entlang zweier unterschiedlicher Muster. Während in der Kindertagesstätte Blau und Gelb stärker betreuerische Aktivitäten wie Essenszubereitung, Händewaschen usw. den Alltag strukturierten, betonten die Kindertagesstätten Rot und Grün stärker den Bildungsaspekt: pädagogische Zielsetzungen und entsprechende Lernaktivitäten für die Kinder. Besonders auffällig im Vergleich der Kitas erschien uns dabei auch die unterschiedliche zeitliche Rhythmisierung des Tagesablaufs (vgl. Tab. 5.1). Waren die zeitlichen Wechsel der Aktivitäten in den Kitas Blau und Gelb auffallend kleinteilig, fanden im Kontrast dazu in den Kitas Rot und Grün sehr lange Zeiten des nicht unterbrochenen freien Spiels statt. Der Vergleich zeigt, dass sich die Kitas erheblich in der Art und Weise unterscheiden, wie alltägliche Aktivitäten organisiert werden, welchen Stellenwert sie im Tagesablauf erhalten und auch

Tab.5.1 Tagesabläufe im kontrastierenden Vergleich zweier Kitas. Die fett gesetzten Begriffe wirken jeweils als Taktgeber

\begin{tabular}{l|l}
\hline Chronos: Kita Blau & Kairos: Kita Grün \\
\hline Ankommen & Ankommen \\
Freispiel/Angebot & Freispiel \\
Kreisspiel & Bis zum gemeinsamen \\
Znüni & Aufbruch in den Wald \\
Freispiel/Angebot & Freispiel \\
Gemeinsam Wickeln & Znüni etc. nach Bedarf \\
Gemeinsam Händewaschen & $\ldots$ \\
Zmittag & $\ldots$ \\
Mittagsschlaf & Zmittag \\
Freispiel/Angebot & Mittagsschlaf \\
Gemeinsam Wickeln & Freispiel \\
Zvieri & Zvieri \\
Abholen & $\ldots$ \\
& Zurück zum Garten, \\
& Freispiel bis zum Abholen \\
\hline
\end{tabular}


hinsichtlich des dabei zur Anwendung kommenden Zeitverständnisses. Ist in Kita Gelb und Blau die exakte Einhaltung zum Beispiel von Essenszeiten zentral, wird hier Chronos, die lineare Uhrzeit, präferiert, ein Zeitverständnis, das die Effektivität und Effizienz der Abläufe betont (Orlikowski und Yates 2002). Anders in den Kitas Rot und Grün, hier sind das individuelle Erleben, das kindliche Spiel und die im Kita-Alltag stattfindenden Lernprozesse zentral und damit Kairos, ein Zeitverständnis, in dem das Erleben in den Mittelpunkt gerückt wird.

Im Folgenden beschreiben wir diese Unterschiede in den zeitlichen Ordnungen der Tagesabläufe genauer anhand unserer ethnografischen Beobachtungen der Rhythmen und Routinen in den einzelnen Kitas.

\subsubsection{Kita Blau und Gelb: Chronos oder der Haushalt als Taktgeber}

In der Kita Gelb haben wir die folgende Routine beobachtet:

Es ist kurz vor der Mittagspause. Die Kinder spielen in der Eingangshalle, die Kinderbetreuerin ist bei ihnen. Maurin, der männliche Auszubildende ${ }^{1}$, deckt den Tisch. Ein Kollege aus einer anderen Gruppe hilft ihm, alles rechtzeitig zu schaffen. Maurin sortiert kleine Symbole, die auf dem Tisch verteilt werden, um den Kindern jeweils einen bestimmten Platz am Tisch zuzuweisen. Alles ist in dieser Situation für die Kinder vorbereitet. Der Salat befindet sich bereits auf den Tellern, das Wasser ist bereits in die Becher gegossen.

Während Maurin Wasser eingießt, findet das folgende In-situ-Interview statt:

Interviewerin: Hat das einen bestimmten Grund, warum ihr alles schon so fix und fertig macht, bevor die Kinder reinkommen?

Maurin: Ähm. Einfach zur Vorbereitung. Weil nachher, wir haben dann nur 20, 25 min zum Essen, halbe Stunde höchstens, und es braucht halt wieder Zeit, wenn man jedem Kind aufgeben muss. (...)

Interviewerin: Warum sind es nur 25 min zum Mittagessen?

Maurin: Weil sie nachher dann schlafen müssen, sie müssen Zähne putzen, dann muss ich halb eins das Geschirr in die Küche geschickt haben, ja, es ist zeitmäßig alles, nicht ein Stress, aber schon knapp, dann muss ich noch putzen, die Zähne putzen mit den Kindern, jetzt habe ich die „Liegi“ noch nicht mal bereit (das Matratzenlager für den Mittagsschlaf, das in einem Zimmer jeweils bereit gemacht wird). Hauptsächlich ist es

\footnotetext{
${ }^{1}$ Hierbei und auch bei allen weiteren in Feldnotizen und Interviewzitaten wiedergegebenen Namen handelt es sich ausschließlich um Pseudonyme.
} 
wegen der Kinder, dass sie nachher schlafen gehen, es ist auch so, dass Kinder schon am Tisch einschlafen, oder so, und, äh, ja. (Video Transkript, Kita Blau)

Maurin verweist in seiner Erklärung auf einen fix etablierten Zeitplan und eine Liste von Dingen, die er in dieser Zeit zu erledigen hat. Die Zeitplanung ist sehr eng getaktet, wie er betont. Das rechtzeitige Zurückbringen des Geschirrs in die Küche der Kita und das Abräumen des Tischs dominieren Maurins Aktivitäten. Die Kinder sind hier nicht Teil der Routine der Nahrungszubereitung für das Mittagessen. Im Mittelpunkt der hier vollzogenen Routine stehen effiziente Abläufe und das Einhalten eines strengen Zeitplans. Was Maurin hier beschreibt, ist das, was wir auch in Kita Gelb beobachtet haben: Die Haushaltsaktivitäten dominieren die Strukturierung der Arbeits- und Tagesabläufe. Effizienz der Abläufe und die Einhaltung der klar festgelegten Uhrzeiten für die Mahlzeiten sind wichtige, die Aktivitäten strukturierende Zielsetzungen. Zeit wird hier linear, als Chronos verstanden.

Die Kleinteiligkeit des Tagesablaufs zeigt sich auch in der von uns beobachteten Artikulationsarbeit der Kinderbetreuenden (Strauss 1988; vgl. Gherardi 2019, S. 15). Für jeden Übergang von einer Aktivität oder Aufgabe zur anderen wird hier jeweils verhandelt, wer im Team was tun wird. Das Sprechen über die Aufgabenverteilung nimmt einen großen Teil der Gesprächszeit insgesamt in Anspruch. Es entsteht jeweils ein Eindruck von großer Geschäftigkeit. Der Tag wird so in winzigste Sequenzen aufgeteilt und es bleiben nur sehr kurze Zeiträume, die den Kindern wie den Betreuenden für freie Aktivitäten zur Verfügung stehen können.

Der Tagesverlauf wird durch die häufigen Wechsel zwischen einzelnen Routinen gestaltet. Die Routinen finden jeweils mit allen Kindern auf möglichst gleiche Weise statt. Sehr oft beobachten wir Kinder, die beim Händewaschen darauf warten, dass andere Kinder ihre Hände waschen, es sobald sie an der Reihe sind selbst tun, um dann erneut zu warten, bis auch alle anderen Kinder ihre Hände gewaschen haben. Händewaschen wird hier zur zentralen Aktivität, sie strukturiert die Zeit und beansprucht die Aufmerksamkeit; andere Aktivitäten haben währenddessen keinen Platz. Auf ähnliche Weise werden beispielsweise Windeln zu einem bestimmten Zeitpunkt gewechselt und nicht dann, wenn es im Einzelfall notwendig wäre. Ähnlich wie beim Händewaschen führt dies zu einem hohen Anteil an Wartezeiten für die Kinder in Kita Blau. Sie warten darauf, dass andere mit etwas fertig werden, auf einer Bank in der Eingangshalle oder im Kreis am Boden sitzend. Insgesamt wirkt das freie Spiel oder andere, aus pädagogischer Bildungsperspektive zentrale Aktivitäten, als eingeklemmt zwischen Mahlzeiten, Schlafen sowie den für die Körperhygiene wichtigen Aktivitäten wie Händewaschen und Windelnwechseln. 
Die meisten Aktivitäten werden in der Gruppe durchgeführt und der Tagesablauf definiert, was wann zu tun ist. Diese grundsätzliche Vorstellung, Dinge gemeinsam, zum im Tagesplan vorgesehenen Zeitpunkt und innerhalb der hierfür als angemessenen Zeitdauer durchzuführen, ist in Kita Gelb noch deutlicher wie das folgende Memo zeigt. Die zeitliche Ordnung wird durch den Kinderbetreuer disziplinarisch durchgesetzt und der Versuch eines Kindes, sich dieser Ordnung zu entziehen, führt schlussendlich zum Konflikt.

Leo wird mehrmals von Michel darauf hingewiesen, dass er auch im Singkreis hinsitzen soll. Leo ist unruhig und schert immer wieder aus dem Kreis aus. Als Leo sich ganz dem Kreis abwendet und mit der Schnecke zu spielen beginnt (und auch nach der Aufforderung Michels, sich wieder in den Kreis zu drehen), steht Michel auf und räumt die Schnecke weg. Leo schreit „Nein“. Michel droht, den Stuhl zu holen. Leo kommt in den Kreis zurück und scheint ziemlich aufgebracht zu sein, weil Michel den Stuhl bereits in Reichweite platziert hat (der Stuhl dient wohl als Disziplinarstrafe, wenn sich die Kinder nicht richtig benehmen, dann müssen sie auf dem Stuhl sitzen). (Video-Memo, Kita Gelb)

Die aufgestellten Regeln müssen sowohl in Kita Gelb als auch in Kita Blau strikt eingehalten werden. Vor allem in Kita Gelb fällt uns auf, dass die Kinder oft ermahnt werden, sich angepasst zu benehmen und nicht laut oder unruhig zu sein.

Die Aktivitäten im Haushalt erscheinen so als weitgehend unabhängig vom Lernen der Kinder. Obwohl Kinder an einigen der hauswirtschaftlichen Tätigkeiten beteiligt werden, wie dem Abräumen des Tischs und dem Stapeln des Geschirrs am Spültisch nach dem Mittagessen oder indem sie in Kita Blau bei verschiedenen Reinigungsarbeiten helfen, werden diese Aufgaben hauptsächlich von den Kinderbetreuenden erledigt. Das Ziel scheint ein effizienter Umgang mit der zur Verfügung stehenden Zeit zu sein, die Dinge sollen schnell erledigt werden. In den in Kita Blau und Kita Gelb beobachteten alltäglichen Rhythmen und Routinen werden Betreuung und Bildung als klar voneinander getrennte Aktivitäten behandelt, was eine Wahrnehmung der beiden Aufgabenbereiche als unterschiedlich und sogar sich gegenseitig widersprechend erzeugt.

Insgesamt erzeugen die alltäglichen Rhythmen und Routinen in Kita Blau und Gelb eine Dominanz der haushälterischen Tätigkeiten, womit der Diskurs der Betreuung im Mittelpunkt steht. Mahlzeiten, Aufräumen nach den Mahlzeiten, Händewaschen und Windelnwechseln sowie verschiedene Gruppenaktivitäten werden von den Betreuenden als wichtig betont, die Zeiten und Abläufe jeweils eingehalten und eingefordert. Es sind diese Aktivitäten, die den Tag strukturieren. 
Zeit wird hier als „Chronos“ verstanden; ihre Linearität und die damit einhergehende Forderung nach Effektivität und Effizienz werden betont (Orlikowski und Yates 2002). Hausarbeiten sind in erster Linie Aufgaben, die von den Kinderbetreuerinnen und Kinderbetreuern erledigt werden. Auch wenn Kinder an diesen Aktivitäten beteiligt sind, werden die Szenen so gehandhabt, dass der effiziente und effektive Umgang mit der Situation im Vordergrund steht. Die in diesen Tätigkeiten steckende Lernaufgabe wird dabei nicht nur übersehen, sondern deutlich zurückgewiesen. Darauf angesprochen erhalten wir häufig die Antwort, dass sich diese Tätigkeiten nicht als Lernaufgabe eignen würden. Der Alltag wird zeitlich durch die hauswirtschaftlichen Tätigkeiten strukturiert, ein Lernen kann in den kurzen Zeiten dazwischen stattfinden. Durch diese Dominanz der hauswirtschaftlichen Aufgaben im täglichen Rhythmus wird so das Lernen marginalisiert: Obwohl viele Lernmöglichkeiten geschaffen werden, können diese nicht genutzt werden, die Priorisierung der Einhaltung des Zeitplans verunmöglicht dies. Interessanterweise haben wir in den Kindertagesstätten Rot und Grün eine andere Art der Organisation von Zeit und Routinen gefunden, die wir im Folgenden beschreiben.

\subsubsection{Kita Rot und Grün: Kairos oder die Lernerfahrung als Taktgeber}

Anders sieht dies in Kita Grün und Rot aus. Die fürsorglich-pflegerischen Betreuungsaufgaben in der Kita werden zwar auch hier geleistet, allerdings scheinen sie mehr in den Hintergrund zu rücken. Das zentrale Ordnungsmoment in den zeitlichen Strukturen ist hier das Schaffen von möglichst großen zeitlichen Freiräumen für das Freispiel der Kinder. Auch hier gibt es eine Ankunftszeit, einen Znüni (zweites Frühstück), ein Mittagessen gefolgt von einer Mittagsruhe, ein Zvieri (Nachmittagsimbiss) und eine Abholzeit. Auch hier gehen die Kinder aufs WC, werden gewickelt und waschen sich die Hände. Diese Aktivitäten stehen aber viel weniger im Vordergrund als das freie Spiel der Kinder. Was wird hier anders gemacht? Die Vorbereitung der Mahlzeiten sowie die Hygieneaktivitäten scheinen hier stärker auf das Wesentliche reduziert, um Platz für längere ununterbrochene Spielsequenzen zu machen. Dies zeigt die folgende Beschreibung der Tagesroutine in Kita Grün:

Die Kinder gehen den Weg zum Waldplatz gemeinsam als Gruppe. Nach der Ankunft dürfen sie frei ihren Platz zum Spielen suchen. Zum Znüni, der in anderen Kitas für alle verbindlichen Zwischenmahlzeit am Vormittag, kommen sie nicht alle zur gleichen Zeit wieder an der Feuerstelle zusammen. Die ersten, die hungrig werden, veranlassen 
die Betreuenden dazu, Äpfel zu schneiden oder Salzstangen auszupacken. Jedes Kind darf einen Apfelschnitz, an einem anderen Tag drei Salzstangen mitnehmen und sie essen, bei Bedarf auch nochmals. Durch das Weglassen einer Gruppensequenz können die Kinder in ihren selbstgewählten Kleingruppen verbleiben und weiter ihren Spielen nachgehen, sie werden nicht unterbrochen. Zum Mittagessen kommen dann alle zusammen, waschen sich die Hände, setzen sich um die Feuerstelle herum und bekommen ihr Essen geschöpft. Die Mittagspause verbringen die kleineren Kinder, die schlafen möchten, in einem kleinen Ästezelt, die größeren gehen wieder zu ihren vorherigen Spielorten und spielen weiter oder suchen sich neue Beschäftigungen. Es wird kein Kind zum Schlafen gezwungen. Der Zvieri, die Zwischenmahlzeit am Nachmittag, wird gemeinsam eingenommen, sie läutet die Rückkehr zur Kita ein. Im Garten der Kita kann bis zum Abholen durch die Eltern wieder frei gespielt werden. (Feldnotizen, Kita Grün)

Auffällig an dieser Beschreibung des Tagesablaufs in Kita Grün ist, dass sowohl die Betreuung, d. h. die Versorgung der Kinder mit Mahlzeiten, das Händewaschen und Wickeln und der Mittagsschlaf, sowie verschiedene Bildungsaspekte in Form von über den Tag verteilt stattfindenden Lernprozessen Raum erhalten. Keines scheint das andere zu dominieren. Der Tag wird hier entlang des Ziels geordnet, möglichst große Zeiteinheiten für freie Spiel- und Lernaktivitäten der Kinder zu schaffen. Zeit wird hier nicht nur linear als Chronos verstanden, sondern auch Kairos, das Erleben einer Situation, findet hier statt (Orlikowski und Yates 2002). Das Erleben des Spiels wird am Vormittag nicht durch Znüni oder einen Singkreis unterbrochen. Der Moment, in dem ein Kind anmeldet, dass es hungrig ist, dient als Moment, um eine Zwischenmahlzeit für diejenigen anzubieten, für die dies in der Situation passt.

Dieser zentrale Grundsatz, möglichst viel Zeit für Lernaktivitäten bereitzustellen, wird von der Kitaleitung in Kita Rot im Interview betont. Die Kinder sollen sich intensiv mit den anderen Kindern in der Gruppe beschäftigen können und mit ihnen interagieren, ohne dass sie fortwährend von den Betreuenden angeleitet werden. Sich vertiefen zu können und die Kinder möglichst für sich spielen zu lassen, sind hier wichtige Grundsätze, die Kairos betonen:

Wir versuchen, ihnen möglichst lange und ungestörte Spieleinheiten zu ermöglichen und bieten wenig von uns aus Aktivitäten an. Also Kreisli machen und so. Das machen wir. Wir sammeln vor dem Mittag zum Beispiel, aber ähm wir merken, wir wollen nicht unseren Sachen, wo wir das Gefühl haben, die wären gut für sie, (und) sie eigentlich stören. In ihrer, dass sie sich vertiefen können in ein Spiel, (das) können sie nur, wenn man sie eben möglichst lange lässt. (Interview, Kitaleiterin, Kita Rot)

Haushälterische Tätigkeiten und auch die Zubereitung der Nahrung werden in Kita Rot und Grün als Lerngelegenheiten verstanden. Während Reto in Kita Grün die 
Kinder am Rüsten des Gemüses oder Schnitzen der Spieße für das Braten über dem Feuer mitarbeiten lässt, wird in Kita Rot einmal pro Woche gemeinsam mit den Kindern das Brot gebacken, das in der Kita zu den Mahlzeiten gegessen wird. Wie die folgende beobachtete Szene zeigt, steht dabei nicht das Produkt und seine effiziente Produktion im Mittelpunkt der Aktivität, sondern die Beschäftigung der Kinder mit dem Brotbacken, dem Kennenlernen der Zutaten, dem Riechen der Zutaten und dem Fühlen des klebrigen Teigs an den Fingern. Wiederum ist es ein Verständnis von Zeit als Kairos, nicht Chronos, das diese Sequenz anleitet.

\begin{abstract}
Zwei Jungen, etwa zwei und drei Jahre alt und ein etwa dreijähriges Mädchen kommen in die Küche. Stefanie besorgt ihnen Schürzen und hilft ihnen beim Anziehen. Dann fragt sie die Kinder: „Was brauchen wir zum Brotbacken?“ Die Kinder sind sehr begierig darauf, ihre Fragen zu beantworten und die Zutaten aufzuzählen. Sie beginnen mit der Zubereitung des Teigs. Die Kinder dürfen der Reihe nach Zutaten in die Schüssel geben. Stefanie nimmt sich Zeit, um allen die Chance zu geben mitzumachen. Sie macht die Kinder auf den Mehlstaub aufmerksam, der sich in der Luft bildet, nachdem das Mehl in die Schüssel gegeben wurde. Die Atmosphäre ist ruhig und konzentriert. Stefanie beginnt zu singen, während sie den Teig bearbeitet, die Kinder hören zuerst zu, kopieren die Bewegung ihrer Hände mit dem Teig auf dem Tisch und singen dann ein bisschen mit. Stefanie schickt ab und zu das Mädchen, das sich immer wieder den Teig von den Händen leckt zum Händewaschen. Alle Kinder sind jetzt damit beschäftigt, kleinere Teile des Teigs zu bearbeiten. Die Kinder sitzen $60 \mathrm{~min}$ am Tisch und bereiten konzentriert den Brotteig vor. (Feldnotizen und Video-Memo, Kita Rot)
\end{abstract}

Die Gestaltung der haushälterischen Aktivität zeigt den in dieser Szene gezeigten Bildungsanspruch. Stefanie benennt die Zutaten und nutzt so die Aktivität zum Aufbau des kindlichen Wortschatzes, die Mengen der Zutaten ermöglichen mathematische Erfahrungen, die Beobachtung des Mehlstaubs naturwissenschaftliche Beobachtungen. Kairos bedeutet, das Bildungspotenzial im Moment, in dem es sich ergibt, zu nutzen. Der Kinderbetreuer in Kita Grün erzählt, wie er spontan eine Lerngelegenheit ins Zentrum des Vormittags rückt und das Mittagessen flexibel anders organisiert, um die Lerngelegenheit zu nutzen:

Ich komme am Morgen und habe ein Repertoire - und das ist: ich bin, ich kann improvisieren und kann spontaner sein, ich bin mal -, ich kam auf dem Weg dahin, in die Kita, und sah es liegen viele Kastanien am Boden, also ging ich Kastanien sammeln, aber bis die Kinder alle angezogen waren, und sie wollten jetzt auch auf dem Weg irgendwie alles anschauen, gut, dann gingen wir dort hin. Ich habe total etwas anderes geplant, am Nachmittag, und das Essen ist auch eingekauft, ging aber, das war zu Fuß ist das fünf Minuten, wir haben sicher zwei Stunden bis dort hin. Also wenn ich laufe, habe ich fünf Minuten, und nicht anderthalb/zwei Stunden, dort Kastanien sammeln und jede Kastanie anschauen, und vergleichen und sortieren, das 
gehört dazu, war es plötzlich Mittag, dann habe ich nur noch gewusst, ja jetzt muss ich Pizzas bestellen [beide lachen], ja ist jetzt vielleicht nicht besonders gut improvisiert, aber vielleicht auch nicht sehr kreativ, aber es ging am schnellsten, und äh - ich mit dem Personal konnte ich das auch sehr gut abdecken. Und haben dann dort Pizzas gegessen auf einem Stein, und ja, sind dann Nachmittag irgendwann wieder zurückgekommen. (Interview, männlicher Kinderbetreuer Reto, Kita Grün)

Die Tagesstruktur ist zwar angelegt, muss aber nicht rigide umgesetzt werden. Die Lerngelegenheit, die sich hier alternativ zum pünktlichen Mittagessen angeboten hat, erhält oberste Priorität und die angelegte zeitliche Struktur muss hier keinesfalls exakt umgesetzt werden. Diese allgemeine Flexibilität spiegelt sich auch im Umgang mit den Kindern wider. Kinderbetreuerinnen und -betreuer revidieren ihre Pläne, sollten sie nicht den Bedürfnissen der Kinder entsprechen. Die Kinder dürfen sich hier in ihren Aktivitäten verlieren und jeweils selbstständig entscheiden, was sie tun wollen. Wir beobachteten in Kita Grün und Rot tatsächlich lange Sequenzen des freien Spiels, die ohne Interventionen durch die Betreuenden verlaufen.

Die langen Freispielsequenzen werden auch durch die Organisation der Übergänge zwischen Aktivitäten ermöglicht. So verlässt zum Beispiel ein Kinderbetreuer in Kita Rot früher als andere den Mittagstisch, geht auf die Toilette und hilft dort den ersten Kindern beim Zähneputzen, Händewaschen und Windelnwechseln. Kinder, die ihre Mahlzeiten beendet haben, können aufstehen, ihr Geschirr wegräumen und ins Badezimmer gehen, um sich für das Spiel oder den Mittagsschlaf vorzubereiten, ohne jeweils auf andere Kinder warten zu müssen. Dasselbe beobachten wir beim Übergang zum Spiel im Garten. Einer der Kinderbetreuer prüft, ob die Tore verschlossen sind und bleibt draußen, um die Kinder in Empfang zu nehmen, die als erste bereit waren, um im Garten zu spielen. Eine zweite Person half den Kindern, ihre Schuhe und Hüte anzuziehen und Sonnencreme aufzutragen. Diese Art der Organisation von Übergängen zwischen Sequenzen ermöglicht es den Kindern, sich in ihrem eigenen Tempo vorzubereiten und nicht fortlaufend auf andere Kinder warten zu müssen. Auffällig ist, dass so auch viel weniger disziplinierende Aktivitäten beobachtet wurden. Diese werden scheinbar unnötig, da die langen und für die Kinder langweiligen Wartezeiten wegfallen.

Auch in den Kitas Rot und Grün gibt es feste zeitliche Abläufe, die dem Tag Struktur geben. In Kita Rot wird beispielsweise der Tagesablauf in einem an der Küchenwand hängenden komplexen Diagramm dargestellt. Der Tag ist in kleinen Sequenzen organisiert. Die Grafik enthält alle Aktivitäten der Betreuungspersonen, wie zum Beispiel Kochen, Windeln wechseln, Zähne putzen, aber auch eine Gruppenaktivität vorbereiten oder ein Teammeeting abhalten. Die Gruppenleiterin Stefanie erklärt im Interview, dass dieser Zeitplan wichtig sei, um die Arbeit der 
Betreuenden zu organisieren und insbesondere den Auszubildenden transparent zu vermitteln, was jeweils zu tun ist. Darüber hinaus übernähmen die Betreuerinnen und Betreuer ihrerseits die Organisation der Aufgaben und Aktivitäten für einen Tag oder einen halben Tag. Aufgrund der transparenten Darstellung werde keine Zeit für die Organisation dieser Tätigkeiten und Abläufe benötigt. Trotz dieses komplexen und bis auf die kleinste Aufgabe detaillierten Plans erweckt der Tagesablauf auf uns als Beobachtende nicht den Eindruck, starr zu sein oder in winzige Mikroaktivitäten aufgeteilt zu werden. Der aufgehängte Tagesplan für die Zuteilung der Aufgaben der Kinderbetreuenden und die klare Zuteilung der Entscheidung für den jeweiligen Tag an ein Teammitglied führen dazu, dass sich die Kinderbetreuenden in Kita Rot während des Tages laufend und häufig absprechen, wie wir es als Artikulationsarbeit in Kita Blau so häufig beobachtet haben. In der täglichen Praxis von Kita Rot erscheint uns die Handhabung des zeitlichen Ablaufs ausgesprochen flexibel gestaltet und am aktuellen Bedarf und damit auf die notwendigen pädagogischen Zielsetzungen ausgerichtet. Beide Kindertagesstätten, Rot und Grün, organisieren die Zeit um einige feste Zeiten herum, wie zum Beispiel das Frühstück mit der Ankunft der Kinder, das Mittagessen mit einer kurzen Gruppenaktivität zu Beginn und einem ruhigeren Teil nach dem Essen. Die üblichen Zwischenverpflegungen am Vormittag und Nachmittag werden nach Möglichkeit als freiwillige Snacks angeboten: Die Kinder werden dann gefragt, ob sie Hunger haben und es werden Obst oder Kekse angeboten. Die in Kita Blau und Gelb zu diesen Zeiten beobachtete Gruppenroutine entfällt hier häufig bewusst. Zudem sind die Kinder oft aktiv an der Vor- oder Zubereitung der Mahlzeiten wie dem Backen von Brot, dem Schneiden von Äpfeln oder Brot beteiligt und lernen so, wie man mit einem Messer umgeht, ohne verletzt zu werden.

Im Gegensatz zur Dominanz des Diskurses der Betreuung in den Kitas Blau und Gelb priorisieren die Kitas Rot und Grün Bildung und Lernen als die wichtigsten Organisationsprinzipien. Obwohl auch hier Betreuung, Hygiene und gesunde Ernährung wichtig sind, dominieren sie nicht die alltäglichen Rhythmen und Routinen. Der Tagesablauf ist so organisiert, dass den Kindern so viel Raum wie möglich für Spiel und andere autonome Aktivitäten zur Verfügung steht. Routinen werden hinsichtlich der unterstützten Lernaktivität der Kinder kritisch reflektiert und häufig reduziert oder der Situation angepasst, sollten sie den pädagogischen Zielen zuwiderlaufen. Im Mittelpunkt steht die Pädagogik und sie ist auch zentral für das hier vermittelte Bild guter Kinderbetreuung.

Für die von uns verfolgte Fragestellung, welche gendered practices mit diesem Bild aktiviert werden, ist festzustellen, dass dies zumeist äußerst widersprüchlich und häufig sogar offen bleibt. Zwar kann ein Brotbacken am Küchentisch, begleitet durch die weibliche Kinderbetreuerin als hauswirtschaftliche Tätigkeit 
und damit als doing femininity interpretiert werden. Ebenso kann das Hacken von Holz zur Vorbereitung des Feuers im Wald, begleitet durch den männlichen Betreuer als doing masculinity verstanden werden. Tatsächlich sind es aber genau diese stereotypen Erwartungen, die in den von uns beobachteten Szenen jeweils enttäuscht werden und uns damit einen Hinweis auf das Stattfinden von undoing gender geben (Hirschauer 1994, 2001). Die Art und Weise, wie die Praktiken umgesetzt werden sowie auch die in den Interviews geäußerten Begründungen für diese Praktiken zeigen, dass Brotbacken und Holzhacken als Lerngelegenheiten genutzt werden. Indem im konkreten Handeln der Fokus auf den Lernprozess der Kinder gerichtet wird, steht nicht Betreuung im Zentrum, sondern Bildung. Es handelt es sich um ein doing pedagogy, bei dem zugleich gender zumindest für diese Episode in den Hintergrund gerückt wurde.

\section{5 (Un)doing gender in Rhythmen und Routinen}

Die Diskurse der Betreuung und Bildung haben sich nicht nur für die geschlechterdifferenzierende Konstruktion des Berufsbilds in historischer Perspektive als relevant erwiesen, sondern scheinen auch für die Organisation der Rhythmen und Routinen im Kita-Alltag als äußerst relevant. Unsere ethnografische Erforschung der beobachtbaren Routinen sowie der Interviews mit Kinderbetreuenden zu den jeweiligen Zielsetzungen in der Gestaltung der Tagesstruktur hat uns erlaubt, die gendered practices des weiblich konnotierten Felds der Kitas herauszuarbeiten. Ein ethnografischer Zugang ist hier wesentlich, da das in die Rhythmen und Routinen eingeschriebene Wissen über gute Kinderbetreuung nicht explizit sprachlich zur Verfügung steht und erst durch Interpretation zugänglich wird.

Zentral für die Erkenntnisgewinnung ist dabei der Vergleich zwischen den Fällen (Vogt 2002b) und unsere Analyse hat grundlegende Unterschiede zwischen jeweils zwei der ethnografisch untersuchten Kitas festgestellt. In allen Kitas gehören Haushaltaktivitäten und Hygiene zum Alltag: Hände waschen, Tisch decken, Mittagessen einnehmen, aufräumen, wickeln und Zwischenmahlzeiten offerieren sind essenzielle Bestandteile der Tagesstruktur. Die Analyse der Art und Weise, wie diese Aktivitäten von den Kinderbetreuenden gestaltet werden, erlaubt Einblicke in die eng mit der Alltagsorganisation verknüpften gendered practices der Kita. In den Kitas Blau und Gelb baut das dominante Organisationsprinzip auf dem Diskurs der Betreuung auf. Organisatorische Rhythmen und Routinen priorisieren ein Verständnis guter Kinderbetreuung, das die Ernährung, Hygiene und Sicherheit der Kinder sowie die Effizienz der Abläufe betont. Zeit wird als Chronos verstanden und das Verhältnis zwischen Betreuen und Bilden ist hier ein 
Gegensätzliches, die Betreuung ist der Bildung immer vorrangig und dominiert diese. Die Betreuenden befinden sich hier unter ständigem Druck, die Regeln und Abläufe genau einzuhalten. Im Unterschied dazu werden in den Kitas Grün und Rot Haushaltsaktivitäten als Lerngelegenheiten gesehen und genutzt, Bildung und Betreuung gehen hier Hand in Hand und unterstützen sich gegenseitig. In diesen Kitas werden lange, ungestörte Zeiteinheiten dem freien Spiel der Kinder gewidmet. Das Erleben und damit verbunden das Lernen stehen hier im Zentrum, Zeit wird als Kairos verstanden. Damit kommt es auch nicht so sehr auf das genaue Einhalten von gemachten Plänen an, Pläne werden flexibel den aktuellen Bedürfnissen angepasst. Die Reflexion dieser Taktgeber erwies sich allerdings in Workshops mit Kinderbetreuenden als herausfordernd, da die organisatorischen Rhythmen und Routinen kaum hinterfragt werden. Gerade weil die unterschiedlichen Idealbilder in der Gestaltung der Rhythmen und Routinen in manchen Kitas so subtil Wirkung entfalten, besteht möglicherweise gerade diesbezüglich ein großes Potenzial für die Veränderung von festgelegten Genderismen.

Mit der unterschiedlichen Prioritätensetzung in der Rhythmisierung des Tagesablaufs werden auch unterschiedliche Idealbilder der guten Kinderbetreuung vermittelt. In den Kindertagesstätten Blau und Gelb fühlen sich die Kinderbetreuenden verpflichtet, Abläufe effizient und geordnet zu gestalten und dabei den hauswirtschaftlichen Tätigkeiten und Anforderungen Priorität einzuräumen. Historisch sind diese Tätigkeiten weiblich konnotiert und eng mit dem Bild der Hausfrau und Mutter verknüpft, weswegen wir dieses Bild als das „Ideal hausfraulicher Weiblichkeit“ bezeichnet haben. Im Gegensatz dazu lässt ein auf die Bildung fokussiertes Idealbild guter Kinderbetreuung die hauswirtschaftliche Weiblichkeit ruhen und stellt Lernprozesse in freien Aktivitäten und im freien Spiel in den Vordergrund. Mitarbeitende in Kita Grün und Rot werden ermutigt, Lernmöglichkeiten zu schaffen, aufmerksam für die Bedürfnisse der Kinder zu sein, die Entwicklung der Kinder zu beobachten und Alltagssituationen in Lernereignisse $\mathrm{zu}$ verwandeln. Hier wird ein Idealbild von Kinderbetreuung als guter Pädagogik vermittelt, das hinsichtlich der damit verbundenen vergeschlechtlichten Konnotationen eine größere Offenheit aufweist, d. h. es kann nicht eindeutig dem einen oder anderen Geschlecht zugewiesen werden. In den organisationalen Rhythmen und Routinen der Kitas findet doing gender in Form einer Dramatisierung einer ganz bestimmten Weiblichkeit, der hauswirtschaftlichen Weiblichkeit statt, während ein undoing gender mit der im Idealbild guter Kinderbetreuung als guter Pädagogik stattfindenden Dethematisierung von Geschlecht einhergeht. 


\section{Literatur}

Acker, Joan (1990). Hierarchies, Jobs, Bodies - A Theory of Gendered Organizations. Gender \& Society, 4(2), 139-158.

Aigner, Josef C., \& Rohrmann, Tim (2012) Einführung: Public Fathers- Männer in pädagogischen Berufen. In Josef C. Aigner, \& Tim Rohrmann (Hrsg.), Elementar - Männer in der pädagogischen Arbeit mit Kindern (S. 17-98). Opladen: Barbara Budrich.

Andres, Beate, \& Laewen, Hans-Joachim (2011). Das Infans-Konzept der Frühpädagogik: Bildung und Erziehung in Kindertagesstätten. Weimar: Verlag das Netz.

Ariès, Philippe (1998). Geschichte der Kindheit (18. Auflage). München: Deutscher Taschenbuchverlag.

Bruni, Attilla, Gherardi, Silvia, \& Poggio, Barbara (2005). Gender and Entrepreneurship: An Ethnographic Approach. Milton Park: Routledge.

Cameron, Claire (2006). Men in the Nursery Revisited: Issues of male workers and professionalism. Contemporary Issues in Early Childhood, 7(1), 68-79.

Cross, Simon, \& Bagilhole, Barbara (2002). Girls' jobs for the boys? Men, masculinity and non-traditional occupations. Gender, Work and Organization, 9(2), 204-226.

Czarniawska, Barbara (2007). Shadowing and Other Techniques for Doing Fieldwork in Modern Societies. Copenhagen: Copenhagen Business School Press.

Eberle, Thomas, \& Maeder, Christoph (2010). Organizational Ethnography. In David Silverman (ed.). Qualitative Research (pp. 53-73). Los Angeles: SAGE Publications Ltd.

Faeh, Andrea, Wenger, Lydia, \& Vogt, Franziska (in Vorb.). Quality beyond Regulations. Country background Report for Switzerland. Bern: Konferenz der kantonalen Sozialdirektorinnen und Sozialdirektoren.

Falk, Francesca (2018). Gender Innovation and Migration in Switzerland. New York, NY: Palgrave Pivot.

Friebertshäuser, Barbara (1997). Sozialpädagogik im Blick der Frauenforschung. BELTZ. Deutscher Studienverlag.

Friis, Pia (2008). Männer im Kindergarten. Wie man sie anwirbt - und dafür sorgt, dass sie auch bleiben. Themenheft des Norwegischen Kultusministeriums (2006). Deutsche Fassung 2008, Hrsg. vom Forschungsprojekt Elementar, Universität Innsbruck. Verfügbar unter https://www.uibk.ac.at/psyko/forschung/elementar/literatur/friis_ maenner_im_kindergarten.pdf [30.06.20].

Gascoigne, Charlotte, Parry, Emma, \& Buchanan, David (2015). Extreme work, gendered work? How extreme jobs and the discourse of «personal choice» perpetuate gender inequality. Organization, 22(4) 457-475.

Geertz, Clifford (1977). The Interpretation of Cultures: Selected Essays. New York: Basic Books.

Gherardi, Silvia (1995). Gender, Symbolism and Organizational Cultures. London: Sage.

Gherardi, Silvia (2019). How to Conduct a Practice-based Study: Problems and Methods. Cheltenham: Edward Elgar.

Glaser, Barney G., \& Strauss, Anselm (1967). The Discovery of Grounded Theory: Strategies for Qualitative Research. Chicago: Aldine Pub.

Gobo, Giampietro (2008). Doing Ethnography. Los Angeles: Sage. 
Godfrey, Richard, Lilley, Simon, \& Brewis, Joanna (2012). Biceps, Bitches and Borgs: Reading Jarhead's Representation of the Construction of the (Masculine) Military Body. Organization Studies, 33(4), 541-562.

Hirschauer, Stefan (1994): Die soziale Fortpflanzung der Zweigeschlechtlichkeit. Kölner Zeitschrift für Soziologie und Sozialpsychologie, 46(4), 668-692.

Hirschauer, Stefan (2001). Das Vergessen des Geschlechts. Zur Praxeologie einer Kategorie sozialer Ordnung. Kölner Zeitschrift für Soziologie und Sozialpsychologie, Sonderheft 41, 208-235.

Kelan, Elisabeth (2008). Gender, Risk and Employment Insecurity: The Masculine Breadwinner Subtext. Human Relations, 61(9), 1171-1202.

Lanfranchi, Andrea, \& Schrottmann, Ria E. (2004). Kinderbetreuung ausser Haus - eine Entwicklungschance. Bern: Haupt Verlag.

Maihofer, Andrea (2004). Was wandelt sich im aktuellen Wandel der Familie? In Joachim Beehorst, Alex Demirovic, \& Michael Guggemos (Hrsg.), Kritische Theorie im gesellschaftlichen Strukturwandel (S. 384-408). Frankfurt a. M.: Suhrkamp.

Martin, Patricia Y. (2003). «Said and Done» Versus «Saying and Doing». Gendering Practices, Practicing Gender at Work. Gender \& Society, 17(3), 342-366.

Mayer, Christine (2009). Macht in Frauenhand. Fallbeispiele zur Berufsbildung im 19. Jahrhundert. In Martina Löw (Hrsg.), Geschlecht und Macht: Analysen zum Spannungsfeld von Arbeit, Bildung und Familie (S. 193-213). Wiesbaden: VS Verlag für Sozialwissenschaften.

Meriläinen, Susan, Tienari, Janne, Thomas, Robin, \& Davies, Annette (2004). Management Consultants Talk: A Cross-Cultural Comparison of Normalizing Discourse and Resistance. Organization, 11(4), 539-564.

Nentwich, Julia C., Poppen, Wiebke, Schälin, Stefanie, \& Vogt, Franziska (2013). The Same and the Other: Male Childcare Workers Managing Identity Dissonance. International Review of Sociology, 23(2), 325-344.

Orlikowski, Wanda J. \& Yates, JoAnne (2002). It's about Time: Temporal Structuring in Organizations. Organization Science, 13(6), 684-700.

Perren, Sonja, Frei, Doris, \& Herrmann, Sandra (2016). Pädagogische Qualität in frühkindlichen Bildungs- und Betreuungseinrichtungen in der Schweiz. Erste Erfahrungen und Befunde mit dem CLASS Toddler Beobachtungsverfahren. Frühe Bildung, 5(1), 3-12.

QualiKita (2014). QualiKita-Handbuch: Standard des Qualitätslabels für Kindertagesstätten. Verband Kindertagesstätten der Schweiz. Jacobs Foundation. Verfügbar unter https:// www.quali-kita.ch/de/fuer-kitas/kostenlose-arbeitsinstrumente/ [20.6.20].

Rabe-Kleberg, Ursula (2003). Gender Mainstreaming und Kindergarten. Weinheim: Beltz.

Rohrmann, Tim (2009). Gender in Kindertageseinrichtungen. Ein Überblick über den Forschungsstand. Deutsches Jugendinstitut: München.

Rossbach, Hans-Günther; \& Grell, Frithjof (2012). Vorschulische Einrichtungen. In Uwe Sandfuchs, Wolfgang Melzer, Bernd Dühlmeier, \& Andy Rausch (Hrsg.), Handbuch Erziehung (S. 332-337). Bad Heilbrunn: Klinkhardt.

Sargent, Paul (2005). The Gendering of Men in Early Childhood Education. Sex Roles, 52(2), 251-259.

Strauss, Anselm (1988). The Articulation of Project Work: An Organizational Process. The Sociological Quarterly, 29(2), 163-178. 
Timmerman, Greetje, \& Schreuder, Pauline (2008). Gendered Constructions of Professionals in Daycare. Sex Roles, 59, 199-213.

Vogt, Franziska (2002a). A Caring Teacher - Explorations into Primary School Teachers' Professional Identity and Ethic of Care. Gender and Education, 14(3), 252-264.

Vogt, Franziska (2002b). No Ethnography Without Comparison: The Methodological Significance of Comparison in Ethnographic Research. In Geoffrey Walford (Ed.), Debates and Developments in Ethnographic Methodology (pp. 23-42). Oxford: JAI/Elsevier Science.

Vogt, Franziska (2015). Bildung in der frühen Kindheit: «Bildung» und «pädagogische Qualität» als Auslöser von Angebotsausbau und Innovation. In Sandra Hupka-Brunner, Hans-Ulrich Grunder, Manfred M. Bergman, Manfred, \& Christian Imdorf (Hrsg.): Qualität in der Bildung (S. 13-27). Bad Heilbrunn: Julius Klinckhardt.

Walter-Laager, Catherine (2015). Erfahrungsfelder und Beobachtungspunkte für den Frühbereich. Zürich: Stadt Zürich. Verfügbar unter https://www.stadt-zuerich.ch/content/dam/ stzh/ssd/Deutsch/Volksschule/dokumente/publikationen_broschueren/fruehfoerderung/ dossier_erfahrungsfelder.pdf [20.6.20].

Warin, Jo (2006). Heavy-Metal Humpty Dumpty: Dissonant Masculinities within the Context of the Nursery. Gender and Education, 18(5), 523-537.

Wustmann Seiler, Corina, \& Simoni, Heidi (2012). Orientierungsrahmen für frühkindliche Bildung, Betreuung und Erziehung in der Schweiz. Erarbeitet vom Marie Meierhofer Institut für das Kind, erstellt im Auftrag der Schweizerischen UNESCO-Kommission und des Netzwerks Kinderbetreuung Schweiz. Zürich. Verfügbar unter https://www.orientier ungsrahmen.ch [30.06.20].

Open Access Dieses Kapitel wird unter der Creative Commons Namensnennung 4.0 International Lizenz (http://creativecommons.org/licenses/by/4.0/deed.de) veröffentlicht, welche die Nutzung, Vervielfältigung, Bearbeitung, Verbreitung und Wiedergabe in jeglichem Medium und Format erlaubt, sofern Sie den/die ursprünglichen Autor(en) und die Quelle ordnungsgemäß nennen, einen Link zur Creative Commons Lizenz beifügen und angeben, ob Änderungen vorgenommen wurden.

Die in diesem Kapitel enthaltenen Bilder und sonstiges Drittmaterial unterliegen ebenfalls der genannten Creative Commons Lizenz, sofern sich aus der Abbildungslegende nichts anderes ergibt. Sofern das betreffende Material nicht unter der genannten Creative Commons Lizenz steht und die betreffende Handlung nicht nach gesetzlichen Vorschriften erlaubt ist, ist für die oben aufgeführten Weiterverwendungen des Materials die Einwilligung des jeweiligen Rechteinhabers einzuholen.

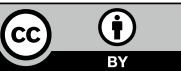

